\title{
The supernova remnant G 6.4-0.1 and its environment
}

\author{
F. Mavromatakis ${ }^{1}$, E. Xilouris ${ }^{2}$, and P. Boumis ${ }^{2}$ \\ 1 University of Crete, Physics Department, PO Box 2208, 71003 Heraklion, Crete, Greece \\ e-mail: fotis@physics.uoc.gr \\ 2 Institute of Astronomy \& Astrophysics, National Observatory of Athens, I. Metaxa \& V. Pavlou, P. Penteli, 15236 Athens, \\ Greece
}

Received 28 April 2004 / Accepted 12 July 2004

\begin{abstract}
Flux calibrated CCD images, in the $\mathrm{H} \alpha+[\mathrm{N} \mathrm{II}]$, [S II], and [O III] emission lines, of a wide field around the supernova remnant G 6.4-0.1 are presented. The low ionization images identify a front of enhanced $\left[\mathrm{S} \mathrm{II}_{3}\right] \mathrm{H} \alpha+[\mathrm{N}$ II] ratio along the eastwest direction. This front is very well correlated with the filamentary radio emission of the remnant as well as with molecular $\mathrm{CO}$ emission and may indicate the interaction of the primary blast wave with molecular clouds present in the vicinity of the remnant. We estimate a total $\mathrm{H} \alpha$ flux, corrected for interstellar extinction, of $2 \times 10^{-8} \mathrm{erg} \mathrm{s}^{-1} \mathrm{~cm}^{-2}$, and a total [S II] flux of $1.1 \times 10^{-8} \mathrm{erg} \mathrm{s}^{-1} \mathrm{~cm}^{-2}$. The $\mathrm{H} \alpha+\left[\mathrm{N}_{\mathrm{II}}\right]$ and $\left[\mathrm{S}_{\mathrm{II}}\right]$ images provide evidence for the presence of emission from shock heated gas to the south-west and to the east of the bulk of the known optical emission, implying that the primary shock wave is able to drive radiative shocks into the interstellar clouds. The image in the medium ionization line of [O III]5007 $\AA$ does not reveal any filamentary structures. On the contrary, the emission is diffuse and very weak, close to our detection limit of $5 \times$ $10^{-17} \mathrm{erg} \mathrm{s}^{-1} \mathrm{~cm}^{-2} \operatorname{arcsec}^{-2}(3 \sigma)$, and appears to be mainly present in the south-east to north-west areas of the remnant. The long-slit spectra indicate significant extinction in all positions observed, while the measured variations are within the $3 \sigma$ error. The [O III] emission in the spectra, whenever present, is weaker than the $\mathrm{H} \beta$ flux suggesting shock velocities around $70 \mathrm{~km} \mathrm{~s}^{-1}$ or less all around the remnant in accordance with the [O III] imagery. Thus, the low shock velocities are a common characteristic of G 6.4-0.1 and not just of the areas where the spectra were acquired. The average sulfur line ratio suggests postshock electron densities below $120 \mathrm{~cm}^{-3}$ at the $3 \sigma$ limit.
\end{abstract}

Key words. ISM: general - ISM: supernova remnants - ISM: individual objects: G 6.4-0.1

\section{Introduction}

It's almost fourty years since the identification of the galactic source G 6.4-0.1 as a supernova remnant (e.g., Kundu 1970, and references therein). This remnant, also known as $\mathrm{W} 28$, lies very close to the galactic plane along with $\mathrm{H}_{\text {II }}$ nebulosities (Jusef-Zadeh et al. 2000; Milne \& Wilson 1971; Shaver \& Goss 1970). Milne \& Hill (1969) determined a non-thermal radio spectral index of -0.4 , while the observations of Kundu (1970) and Milne \& Wilson (1971) showed the presence of polarized emission at the level of a few percent. Long et al. (1991) used Einstein data to study the X-ray morphology of G 6.4-0.1 as well as its spectral properties. The X-ray emission is bounded by the radio emission and is diffuse peaking near the center of the remnant and near its north-east boundary. A detailed spectral study based on data from the ROSAT and ASCA satellites has been presented by Rho \& Borkowski (2002). The overall X-ray emission is thermal but spectral variations are observed. The southwestern shell is characterized by a higher temperature $(\sim 1.5 \mathrm{keV})$ relative to the temperature of $\sim 0.6 \mathrm{keV}$ measured in the northeastern shell. Furthermore, the central emission cannot be fit by single temperature models. Instead, two temperatures of 0.6 and $1.8 \mathrm{keV}$ are required. The long ionization time scales derived from the spectral fits indicate that the gas is close to ionization equilibrium. It is believed that a radiative shell may have formed in the north, while the lower ambient density in the south allows for the application of the Sedov-Taylor solution.

Observations in molecular wavelengths revealed considerable amounts of radiation in the east, north-east areas of G 6.4-0.1 (Arikawa et al. 1999). In addition, the OH masers which have been detected by Claussen et al. (1997) lie preferentially along filaments of shocked gas (Frail \& Mitchell 1998). It is believed that in those locations the supernova remnant interacts with molecular clouds. Additional interest in G 6.4-0.1 is raised by the $0.4 \mathrm{~s}$ period pulsar PSR B1758-23 which lies at the north boundary of the remnant (e.g., Kaspi et al. 1993), while a high energy EGRET source is present in the north-east (Esposito et al. 1996). However, the relation of these sources to G 6.4-0.1 is not clear.

Optical studies of W 28 consist of wide field imaging (e.g., van den Bergh 1973), narrow field $\left(15^{\prime} \times 15^{\prime}\right)$ imaging of selected areas in $\mathrm{H} \alpha$ and [S $\mathrm{SI}$ (Long et al. 1991), and long-slit spectroscopy (Long et al. 1991; Bohigas et al. 1983; Dopita et al. 1977). The optical emission appears mainly diffuse and 
Table 1. Journal of the observations.

\begin{tabular}{lllll}
\hline \hline & $\lambda_{\mathrm{C}}$ & $\Delta \lambda$ & Exp. times $^{a}$ \\
Filter & $(\AA)$ & $(\AA)$ & Date (UT) & 1 No. of frames $\left.{ }^{b}\right)$ \\
\hline $\mathrm{H} \alpha+\left[\mathrm{N}_{\text {II }}\right]$ & 6560 & 75 & $09,12-13 / 08 / 2002$ & $12000(5)$ \\
{$\left[\mathrm{S}_{\mathrm{II}}\right]$} & 6708 & 20 & $09-10,12-13 / 08 / 2002$ & $12000(5)$ \\
{$\left[\mathrm{O}_{\text {III }}\right]$} & 5005 & 28 & $11 / 08 / 2002,20 / 06 / 2004$ & $9600(4)$ \\
\hline
\end{tabular}

${ }^{a}$ Total exposure times in $\mathrm{s}$.

${ }^{b}$ Number of individual frames.

patchy and is confined by the radio emission. A few strong H II nebulosities are projected in the field of the remnant as well as dark regions. The optical spectra point to low electron densities, low shock velocities, and sufficient interstellar attenuation. In this work we present the first flux calibrated images of a wide field centered on $\mathrm{G}$ 6.4-0.1 in $\mathrm{H} \alpha+\left[\mathrm{N} \mathrm{II}_{\text {II }}\right.$, [S II] and [O III] along with high quality long-slit spectra at several locations of the remnant. Information concerning the observations and the reduction of the data is given in Sect. 2, while in Sects. 3 and 4 we present in detail the results of the current imaging and spectral observations. In Sect. 5 we compare the optical data with observations performed in other wavelengths. Finally, in Sect. 6 we discuss the properties of the remnant and its environment and in Sect. 7 we summarize our results.

\section{Observations}

\subsection{Optical images}

The wide field images of W 28 were obtained with the $0.3 \mathrm{~m}$ Schmidt-Cassegrain telescope at Skinakas Observatory, Crete, Greece, from August 9 to August 13, 2002 and on June 20, 2004. A $1024 \times 1024$ Thomson CCD was mounted on the telescope allowing us to observe a $70^{\prime} \times 70^{\prime}$ field with the moderate angular resolution of $\sim 4^{\prime \prime}$ per pixel. All data frames were projected to the same origin of the sky before any arithmetic operations. The astrometric solutions were calculated with the aid of the HST Guide Star Catalogue (Lasker et al. 1999) and all coordinates quoted in this work refer to epoch 2000. A journal of the observations together with information about the filters are given in Table 1. Data reduction was performed with the use of standard IRAF and MIDAS routines (more details can be found in Mavromatakis et al. 2003). The spectrophotometric standard stars HR 5501, HR 7596, HR 7950 and HR 9087 were observed in order to obtain absolute flux calibration (Hamuy et al. 1992, 1994).

\subsection{Optical spectra}

The long-slit spectra of G 6.4-0.1 were obtained on July 10, 2002, and July 30 through August 2, 2003 with the $1.3 \mathrm{~m}$ Ritchey-Cretien telescope at Skinakas Observatory. The hardware setup consisted of a 1300 line $\mathrm{mm}^{-1}$ grating and a SITe $2000 \times 800$ CCD covering the range of 4750-6815 The projected angular dimensions of the slit on the sky are 7 '. $7 \times 7$ '.9 along right ascension and declination, respectively.
Table 2. Spectral log.

\begin{tabular}{llll}
\hline \hline Slit centers & & Total exposure & Slit and aperture \\
\hline$\alpha$ & $\delta$ & time $(\mathrm{s})$ & positions \\
\hline $18^{\mathrm{h}} 01^{\mathrm{m}} 38^{\mathrm{s}}$ & $-23^{\circ} 10^{\prime} 16^{\prime \prime}$ & $7800\left(2^{a}\right)$ & $\mathrm{I},\left[-160^{\prime \prime}, 24^{\prime \prime}\right]^{b}$ \\
\hline $18^{\mathrm{h}} 00^{\mathrm{m}} 44^{\mathrm{s}}$ & $-23^{\circ} 37^{\prime} 12^{\prime \prime}$ & $3900\left(1^{a}\right)$ & $\mathrm{II},\left[8^{\prime \prime}, 26^{\prime \prime}\right]^{b}$ \\
\hline $17^{\mathrm{h}} 59^{\mathrm{m}} 41^{\mathrm{s}}$ & $-23^{\circ} 12^{\prime} 57^{\prime \prime}$ & $7200\left(2^{a}\right)$ & $\mathrm{III},\left[-92^{\prime \prime}, 31^{\prime \prime}\right]^{b}$ \\
\hline $18^{\mathrm{h}} 00^{\mathrm{m}} 04^{\mathrm{s}}$ & $-23^{\circ} 17^{\prime} 29^{\prime \prime}$ & $7200\left(2^{a}\right)$ & $\mathrm{IV},\left[-153^{\prime \prime}, 24^{\prime \prime}\right]^{b}$ \\
& & & $\mathrm{IVa},\left[-32^{\prime \prime}, 22^{\prime \prime}\right]^{b}$ \\
\hline $18^{\mathrm{h}} 00^{\mathrm{m}} 59^{\mathrm{s}}$ & $-23^{\circ} 22^{\prime} 10^{\prime \prime}$ & $7200\left(2^{a}\right)$ & $\mathrm{V},\left[-91^{\prime \prime}, 41^{\prime \prime}\right]^{b}$ \\
& & & $\mathrm{Va},\left[97^{\prime \prime}, 17^{\prime \prime}\right]^{b}$ \\
\hline
\end{tabular}

${ }^{a}$ Number of individual frames.

${ }^{b}$ Spatial offset from slit center and extracted aperture length, both in arcsec.

Coordinates of the slit centers, number of frames and exposure times are given in Table 2. In order to obtain absolute flux calibration of the source spectra the spectrophotometric standard stars HR 5501, HR 7596, HR 9087, HR 718 and HR 7950 were observed.

\section{The optical emission}

\subsection{The $H \alpha+[N I]$, [S II], and [O III] line images}

The low ionization image shown in Fig. 1 reveals the $\mathrm{H} \alpha$ and [N II] 6548, $6584 \AA$ emission in the field of the remnant. The Trifid nebula (M 20), in the north-east edge of our field of view, is a bright $\mathrm{H}$ II region, while the Lagoon nebula (M 8) lies in the south-east. In the south, south-west the bright B9V star HD 163955 (also known as 4 Sgr) is overexposed in all long exposure data frames.

The morphology of the emission is diffuse and patchy and is mainly concetrated in the central areas of G 6.4-0.1 (see also Long et al. 1991). Some structures are seen towards the northeast boundary, while in the north the emission is diffuse and fainter. The flux of $\sim 230 \times 10^{-17} \mathrm{erg} \mathrm{s}^{-1} \mathrm{~cm}^{-2} \operatorname{arcsec}^{-2}$ from the brightest patch of emission is, at least, a factor of 10 lower than the flux measured in the central areas of M 20. The bright structure in the south-east shaped like a " 6 ", at $\alpha \simeq 18^{\mathrm{h}} 02^{\mathrm{m}}$ and $\delta \simeq-23^{\circ} 41^{\prime}$, as well as the filamentary structures in this area do not appear related to $\mathrm{G} 6.4-0.1$ and they may be related to the Lagoon nebula or be simply projected in this field.

The emission detected through the sulfur filter is shown in Fig. 2 along with the radio emission at $1.4 \mathrm{GHz}$ (Dubner et al. 2000). The sulfur emission from the known H II regions in the field is significantly suppressed as expected from photoionized nebulae. The morphology of the remnant in the [S II] filter is indeed similar to that of the $\mathrm{H} \alpha+\left[\mathrm{N}_{\mathrm{II}}\right]$ image as Long et al. (1991) have noted for their fields. However, a major difference concerns the locations of the brightest patches of emission in the two images. The circle in Fig. 1 marks an area which displays a different morphology in the $[\mathrm{S} \mathrm{II}]$ image. Such differences suggest that variations in the $\left[\mathrm{S}_{\mathrm{II}}\right] / \mathrm{H} \alpha$ ratio should exist.

In order to study them in more detail, we take advantage of the fact that the images are flux calibrated and estimate the $\left[\mathrm{S}_{\mathrm{II}}\right] / \mathrm{H} \alpha$ ratio per pixel. However, the calculation of the 


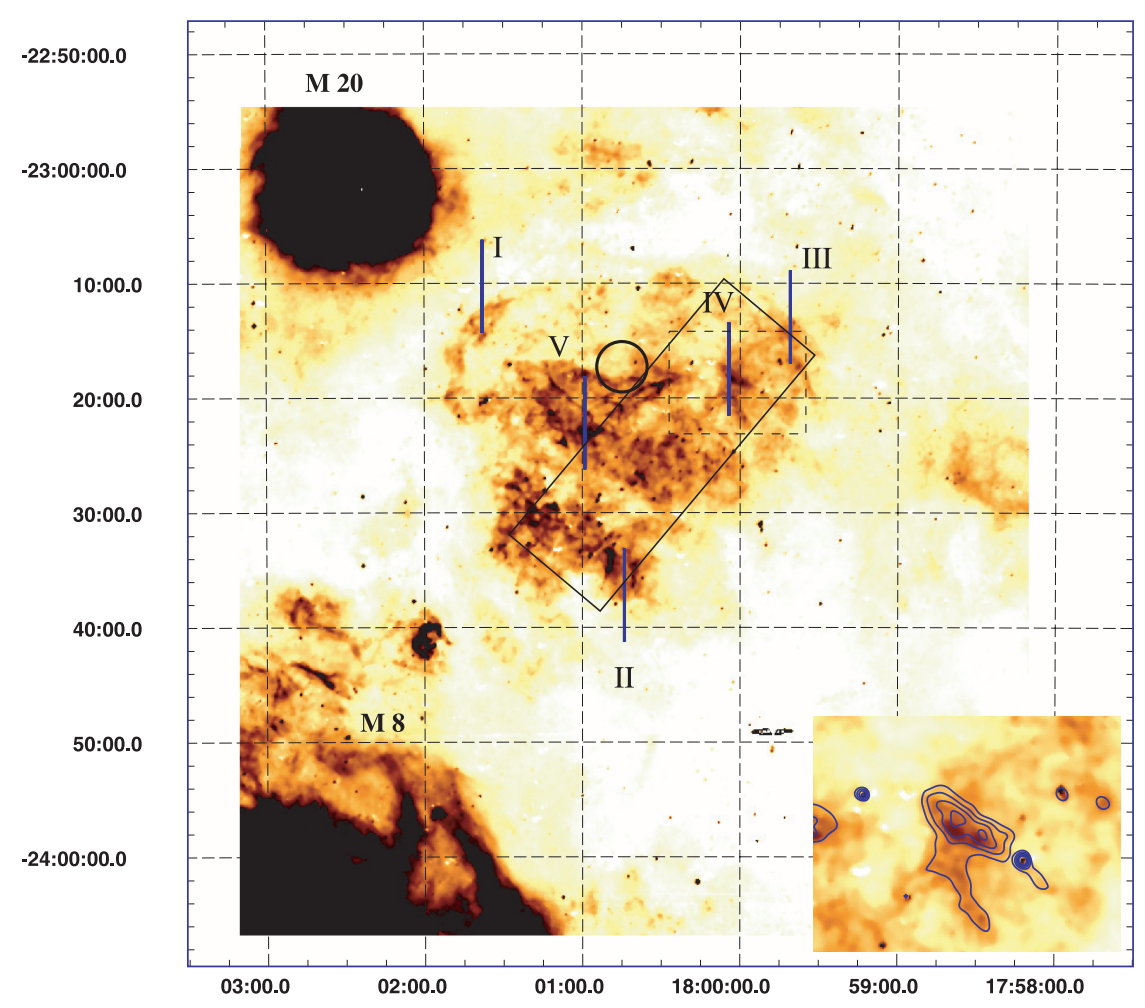

Fig. 1. The field of $\mathrm{G} 6.4-0.1$ in the $\mathrm{H} \alpha+\left[\mathrm{N}_{\text {II }}\right]$ filter. The circle at the center indicates an area displaying different morphology in the [S II] image (Fig. 2), while the long rectangles mark the projections of the slits on the sky. A polygon is drawn to identify the area of weak [O III] emission (see Sect. 3.2), while the dashed rectangle in the north-west outlines the area shown in the inset. The inset $\left(9^{\prime} \times 12^{\prime}\right)$ reveals an offset of $\sim 25^{\prime \prime}$ between the strongest $\mathrm{H} \alpha+\left[\mathrm{N}\right.$ II] flux and the strongest [ $\left.\mathrm{S}_{\mathrm{II}}\right]$ flux. The image has been smoothed to suppress the residuals from the imperfect continuum subtraction, while the shadings run linearly from 0 to $250 \times 10^{-17} \mathrm{erg} \mathrm{s}^{-1} \mathrm{~cm}^{-2} \operatorname{arcsec}^{-2}$.

[S II]/H $\alpha$ image requires knowledge of the filter transmission curves and assumptions about the relative intensity of the [ $\left.\mathrm{N}_{\text {II }}\right]$ flux relative to $\mathrm{H} \alpha$, and the relative strengths of the two sulfur lines. In supernova remnants the $\left[\mathrm{N}_{\mathrm{II}}\right]$ lines are as strong as the $\mathrm{H} \alpha$ emission and we adopt a value of $\sim 1$ for the $[\mathrm{N}$ II $] / \mathrm{H} \alpha$ ratio (Table 3; see also e.g., Raymond et al. 1988; Fesen \& Kirshner 1980), while this ratio drops below 0.6 in H II regions (e.g., Hunter et al. 1992; Fesen \& Hurford 1995). We also adopt the average value of 1.33 for the ratio of the fluxes of the [S II] 6716, $6731 \AA$ lines (Table 3). Finally, the [S II] filter used in the observations transmits $100 \%$ of the $6716 \AA$ line and $18 \%$ of the $6731 \AA$ line and with this data in hand we can proceed to estimate the actual $\left[\mathrm{S}_{\mathrm{II}}\right] / \mathrm{H} \alpha$ ratio as $\sim 3([\mathrm{~S} \text { II }] / \mathrm{H} \alpha+[\mathrm{N} \mathrm{II}])_{\text {observed }}$. This relation provides useful estimates in case of shock heated gas, while it overestimates the ratio for $\mathrm{H}_{\text {II }}$ regions since in photoionized gas the nitrogen lines can be a factor of 2 weaker than the $\mathrm{H} \alpha$ line. The $\left[\mathrm{S}_{\mathrm{II}}\right] / \mathrm{H} \alpha$ ratio, in known SNRs, is typically greater than 0.4 and mostly above 0.5 , (e.g., Smith et al. 1993; Raymond et al. 1988), while in H II regions is found below 0.35 , typically around 0.2 (e.g., Hunter et al. 1992). The M 20 and M 8 nebulae offer the possibility to check this approach. In M 8 the values are close to $\sim 0.3$, while the measurements made over M 20 show variations from $\sim 0.1$ in the center to $\sim 0.25$ in its outer areas. Over areas of emission from the remnant, the ratio image yields typical values in the range of 0.6 to 1.1 .
We can now try to estimate the total $\mathrm{H} \alpha$ and $\left[\mathrm{S}_{\mathrm{II}}\right]$ fluxes since the images are flux calibrated. The same assumptions, as in the derivation of the $[\mathrm{S} \mathrm{II}] / \mathrm{H} \alpha$ ratio image, are made about the $\left[\mathrm{N}_{\text {III }}\right] / \mathrm{H} \alpha$ and $\left[\mathrm{S}_{\text {II }}\right] 6716,6731 \AA$ flux ratios, while the single value of 1.7 , equivalent to $E(B-V)=1.1$, was adopted for the logarithmic interstellar extinction. Integration over the area of the remnant yields an $\mathrm{H} \alpha$ flux of $2 \times 10^{-8} \mathrm{erg} \mathrm{s}^{-1} \mathrm{~cm}^{-2}$ and a [S II] flux of $1.1 \times 10^{-8} \mathrm{erg} \mathrm{s}^{-1} \mathrm{~cm}^{-2}$, corresponding to luminosities, at $1.8 \mathrm{kpc}$, of $7.3 \times 10^{36} \mathrm{erg} \mathrm{s}^{-1}$ and $4 \times 10^{36} \mathrm{erg} \mathrm{s}^{-1}$, respectively. Long et al. (1991) based on their spectra and imagery estimated an $\mathrm{H} \alpha$ flux of $1.3 \times 10^{-8} \mathrm{erg} \mathrm{s}^{-1} \mathrm{~cm}^{-2}$. We consider that the agreement is fair given their limited spatial coverage, and the assumptions made about the distribution of the $\mathrm{H} \alpha$ gas, the central flux, etc.

In Fig. 5 (left) the high resolution radio $1.4 \mathrm{GHz}$ image (Dubner et al. 2000) is shown along with contours of the ratio $\left[\mathrm{S}_{\mathrm{II}}\right] / \mathrm{H} \alpha+\left[\mathrm{N}_{\text {II }}\right]$ image. The contour levels were chosen to point out the highest values of the ratio, while the crosses outline the molecular CO emission (Arikawa et al. 1999). The presence of a front of higher $\left[\mathrm{S}_{\mathrm{II}}\right] / \mathrm{H} \alpha+\left[\mathrm{N}_{\mathrm{II}}\right]$ ratio running roughly in the east-west direction clearly stands out in this figure and verifies the morphological differences reported earlier in this section. Strong sulfur emission is also observed in the east, oriented in the south-north direction, matching rather well the strong radio emission. The spatial position of the front, in the central areas of the remnant, appears very well correlated with the 


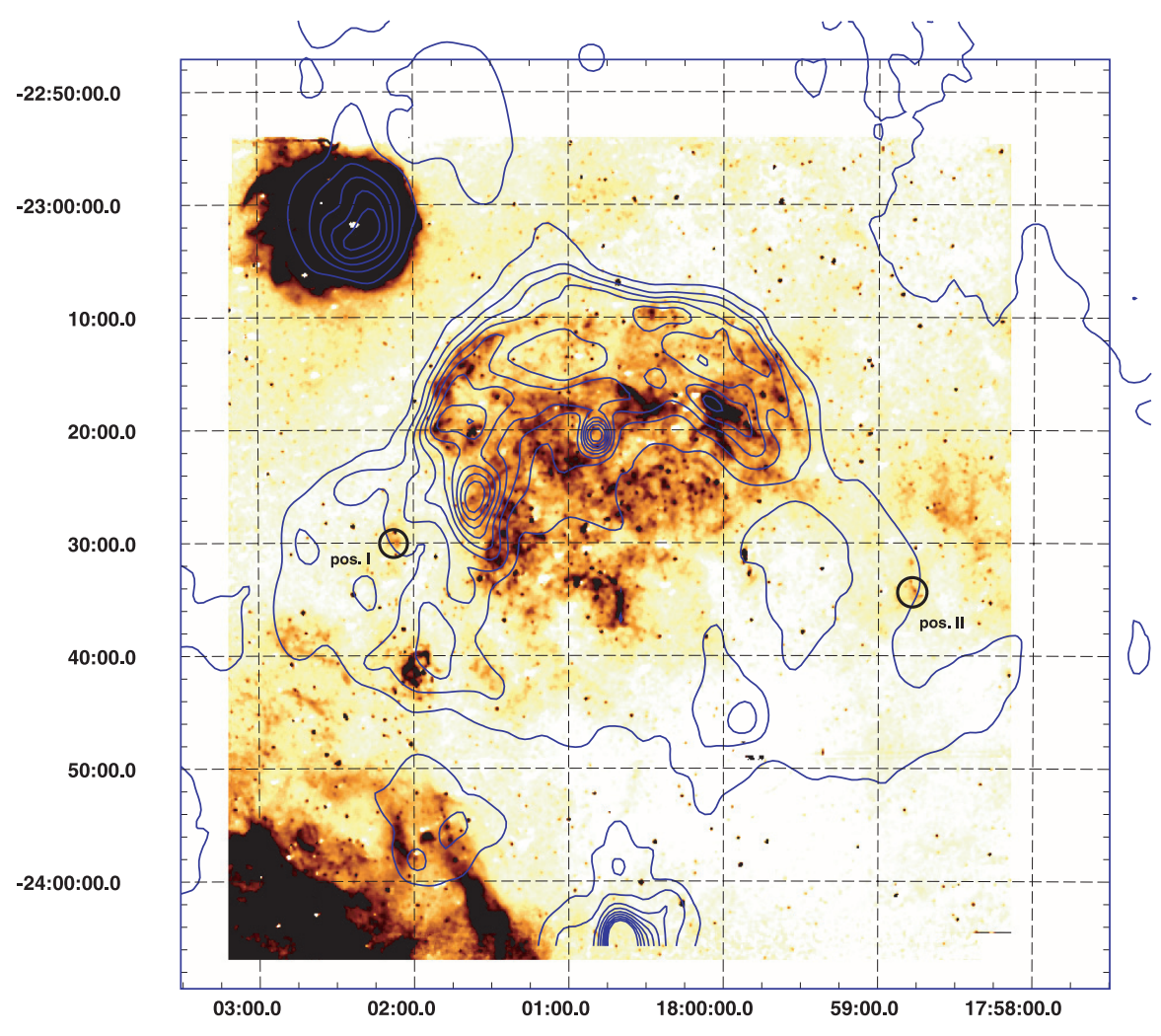

Fig. 2. The field of G 6.4-0.1 in the [S II] filter. The image has been smoothed to suppress the residuals from the imperfect continuum subtraction. The shadings run linearly from 0 to $40 \times 10^{-17} \mathrm{erg} \mathrm{s}^{-1} \mathrm{~cm}^{-2} \operatorname{arcsec}^{-2}$, while the $1400 \mathrm{MHz}$ contours also scale, in a linear fashion, from 0.01 to $1.0 \mathrm{Jy} \mathrm{beam}^{-1}$, every $0.11 \mathrm{Jy} \mathrm{beam}^{-1}$. The small circles labeled pos. I and II mark emission which may originate from shock heated gas. The line segments seen near over-exposed stars are due to the blooming effect.

intense radio emission as well as with the molecular $\mathrm{CO}$ emission (Arikawa et al. 1999). The $\left[\mathrm{S}_{\mathrm{II}}\right] / \mathrm{H} \alpha+[\mathrm{N}$ II] ratio measured along the front is typically twice that measured in other areas of the remnant. The typical projected angular thickness of this front in the center of G 6.4-0.1 is $\sim 20^{\prime \prime}$, equivalent to $\sim 0.2 \mathrm{pc}$ at a distance of $1.8 \mathrm{kpc}$, which is very close or even equal to the dimension of the molecular emission. Further to the west, around $\alpha \simeq 18^{\mathrm{h}} 00^{\mathrm{m}}$ and $\delta \simeq-23^{\circ} 18^{\prime}$, we find a displacement of $\sim 25^{\prime \prime}$ between the strong $\mathrm{H} \alpha+[\mathrm{N}$ II] emission and maxima of the $\left[\mathrm{S}_{\mathrm{II}}\right] / \mathrm{H} \alpha+[\mathrm{N}$ II $]$ ratio. This is clearly shown in the inset of Fig. 1 and it implies that there is a significant spatial offset between areas of strong [S II] and $\mathrm{H} \alpha+\left[\mathrm{N}_{\text {II }}\right]$ emission.

The emission from G 6.4-0.1 in the [O III]5007 $\AA$ line is very weak (Fig. 3). Diffuse emission is present along the southeast to north-west direction but its intensity lies close to the detection limit. We estimate that the [O III] flux is a factor of $\sim 40$ lower than the bright $\mathrm{H} \alpha+[\mathrm{N}$ II] emission measured in the central areas of the remnant. Thus, we expect that the [O III] flux is intrinsically a factor of $\sim 10$ lower than the $\mathrm{H} \alpha+\left[\mathrm{N}_{\text {II }}\right]$ flux since interstellar extinction can account for a factor of 4 (see Sect. 4). Although the diffuse [O III] emission is most likely associated to the remnant as the long-slit spectra suggest, the image is not shown due to the faintness of the emission. The polygon drawn in Fig. 1 shows the approximate boundaries of the weak [O III] emission. The $\mathrm{H}_{\text {II }}$ regions $\mathrm{M} 20$ and $\mathrm{M} 8$ are clearly detected in this medium ionization line.

\section{The long-slit spectra from G 6.4-0.1}

The positions of the long-slit spectra have been determined mainly by two conditions. The first is to provide a satisfactory spatial coverage, while second meets the need for sufficiently good statistics. The projections of the slits on the sky are shown as long, narrow rectangles in Fig. 1. The measured fluxes are given in Table 3 along with some physical quantities than can be deduced from the data, while typical single frame spectra are shown in Fig. 4. Slit location numbers range from I to V, while the spectra designated as IV, IVa and V, Va identify two different aperture spectra extracted from the same frame at positions IV and $\mathrm{V}$, respectively.

The [O I $6300 \AA$ line is generally weak and given the strong night sky in this line, we cannot draw secure conclusions about shock conditions based on this line. The medium ionization line of [O III] $5007 \AA$ is present in some spectra, although weak, while the measured $\mathrm{H} \beta$ fluxes are typically $\sim 11$ times weaker than the $\mathrm{H} \alpha$ fluxes suggesting significant interstellar attenuation of the optical emission. Although significant, the interstellar extinction cannot account for the low [O III] intensity since it could only reduce its flux by a factor of 4 or less with respect to $\mathrm{H} \alpha$.

The electron densities in the cooling zone are low as the ratio of the sulfur lines approaches the high end of the allowable range. The average sulfur line ratio is $1.33( \pm 0.01)$ is equivalent to electron densities of $90( \pm 10) \mathrm{cm}^{-3}$. Thus, electron 
Table 3. Relative line fluxes.

\begin{tabular}{|c|c|c|c|c|c|c|c|}
\hline & $\mathrm{I}(\mathrm{NE})$ & II (S) & III (NW) & IV $(\mathrm{CW})$ & $\mathrm{IVa}(\mathrm{CW})$ & $\mathrm{V}(\mathrm{CE})$ & $\mathrm{Va}(\mathrm{CE})$ \\
\hline Line $(\AA)$ & $F^{1,2}$ & $F^{1,2}$ & $F^{1,2}$ & $F^{1,2}$ & $F^{1,2}$ & $F^{1,2}$ & $F^{1,2}$ \\
\hline $4861 \mathrm{H} \beta$ & - & $8(4)$ & $8(12)$ & $12(8)$ & $8(7)$ & $10(20)$ & $9(8)$ \\
\hline 5007 [O III] & $5(2)$ & - & $5(8)$ & $3(2)$ & - & $3(7)$ & $2(2)$ \\
\hline $5200\left[\mathrm{~N}_{\mathrm{I}}\right]$ & - & $5(4)$ & - & $2(3)$ & $10(14)$ & - & - \\
\hline $6300\left[\mathrm{O}_{\mathrm{I}}\right]$ & $14(9)$ & $12(13)$ & $8(13)$ & $19(19)$ & $41(51)$ & $18(61)$ & $5(7)$ \\
\hline $6364\left[\mathrm{O}_{\mathrm{I}}\right]$ & $5(3)$ & $5(5)$ & $2(4)$ & $5(5)$ & $13(18)$ & $5(15)$ & $2(1)$ \\
\hline $6548[\mathrm{~N} \mathrm{II}]$ & $34(21)$ & $16(18)$ & $25(44)$ & $29(29)$ & $38(43)$ & $17(54)$ & $19(26)$ \\
\hline $6563 \mathrm{H} \alpha$ & $100(58)$ & $100(94)$ & $100(167)$ & $100(90)$ & $100(110)$ & $100(204)$ & $100(129)$ \\
\hline $6584[\mathrm{~N} \mathrm{II}]$ & $113(66)$ & $53(51)$ & $78(135)$ & $93(92)$ & $123(137)$ & $54(159)$ & $60(79)$ \\
\hline $6716[\mathrm{~S} \mathrm{II}]$ & $66(46)$ & $47(45)$ & $49(89)$ & $62(64)$ & $116(132)$ & $29(93)$ & $34(48)$ \\
\hline $6731[\mathrm{~S}$ II] & $49(35)$ & $36(35)$ & $37(68)$ & $47(49)$ & $87(99)$ & $22(69)$ & $25(36)$ \\
\hline Absolute $\mathrm{H} \alpha$ flux ${ }^{3}$ & 31 & 60 & 66 & 50 & 62 & 48 & 67 \\
\hline $\mathrm{H} \alpha / \mathrm{H} \beta$ & - & $13(4)$ & $12(12)$ & $8.2(8)$ & $12(7)$ & $10(20)$ & $11(8)$ \\
\hline$\left[\mathrm{N}_{\mathrm{I}}\right] / \mathrm{H} \beta$ & - & $0.6(3)$ & - & $0.2(3)$ & $1.3(6)$ & - & - \\
\hline$\left[\mathrm{N}_{\mathrm{II}}\right] / \mathrm{H} \alpha$ & $1.5(42)$ & $0.7(46)$ & $1.03(100)$ & $1.2(62)$ & $1.6(83)$ & $0.7(122)$ & $0.8(65)$ \\
\hline$\left[\mathrm{S}_{\mathrm{II}}\right] / \mathrm{H} \alpha$ & $1.15(45)$ & $0.89(49)$ & $0.86(93)$ & $1.09(60)$ & $2.03(91)$ & $0.51(107)$ & $0.59(54)$ \\
\hline $\mathrm{I}(6716) / \mathrm{I}(6731)$ & $1.35(28)$ & $1.31(28)$ & $1.32(54)$ & $1.32(39)$ & $1.33(79)$ & $1.32(55)$ & $1.36(29)$ \\
\hline$c^{4}$ & - & $1.9[0.3]$ & $1.9[0.1]$ & $1.3[0.2]$ & $1.9[0.2]$ & $1.58[0.07]$ & $1.7[0.2]$ \\
\hline$E(B-V)$ & - & $1.3[0.2]$ & $1.26[0.07]$ & $0.9[0.1]$ & $1.3[0.1]$ & $1.05[0.05]$ & $1.1[0.1]$ \\
\hline$n_{\mathrm{e}}\left(\mathrm{cm}^{-3}\right)$ & $70 \pm 50$ & $110 \pm 50$ & $100 \pm 20$ & $100 \pm 30$ & $90 \pm 20$ & $100 \pm 20$ & $60_{-40}^{+50}$ \\
\hline
\end{tabular}

\footnotetext{
${ }^{1}$ Uncorrected for interstellar extinction.

${ }^{2}$ Listed fluxes are a signal to noise weighted average of the individual fluxes.

${ }^{3}$ In units of $10^{-17} \mathrm{erg} \mathrm{s}^{-1} \mathrm{~cm}^{-2} \operatorname{arcsec}^{-2}$.

${ }^{4}$ The logarithmic extinction is determined as $c=1 / 0.331 \cdot \log \left((\mathrm{H} \alpha / \mathrm{H} \beta)_{\mathrm{obs}} / 3\right)$.

Numbers in parentheses represent the signal to noise ratio of the quoted fluxes.

The $1 \sigma$ error is given in square brackets for the logarithmic extinction.

All fluxes are normalized to $F(\mathrm{H} \alpha)=100$.
}

densities as low as $\sim 60 \mathrm{~cm}^{-3}$ and as high as $120 \mathrm{~cm}^{-3}$ are probable at the limit of $3 \sigma$. All electron density calculations were performed with the nebular package as described by Shaw \& Dufour (1995). The weak to vanishing [O III] flux can be explained by slow shocks ( $70 \mathrm{~km} \mathrm{~s}^{-1}$ or less) driven into the interstellar clouds since velocities higher than $\sim 75 \mathrm{~km} \mathrm{~s}^{-1}$ would allow the production of sufficient [O III] $5007 \AA$ to be detected. Such low velocities can also account for the generally weak neutral oxygen emission at $6300 \AA$.

The $\mathrm{H} \alpha / \mathrm{H} \beta$ ratio ranges from the minimum of 8.2 to the maximum of 13.2. However, given the signal to noise of the individual measurements, it is found that the observed differences are in the range of the $3 \sigma$ limit. Consequently, we cannot formally claim the detection of variations in extinction, intrinsic to the remnant based on the current data. In any case, the maximum difference in the color excess that results from a comparison of the minimum and maximum observed Balmer decrements is $0.4( \pm 0.2)$. The individual $E(B-V)$ values and their associated one sigma errors are given in Table 3 . The high resolution $\mathrm{MSX}^{1}$ data at $8.28 \mu \mathrm{m}$ were examined but we could not identify any strong correlation between dust emissivity and the possible variations in the $\mathrm{H} \alpha / \mathrm{H} \beta$ ratio.

\footnotetext{
1 The MSX data were retrieved from the NASA/IPAC Infrared science archive at http://irsa.ipac.caltech.edu
}

\section{Discussion}

The first optical study of the remnant was performed by Long et al. (1991) who obtained $\mathrm{H} \alpha+\left[\mathrm{NII}_{\mathrm{II}}\right.$ and [S II] images of three fields $\left(15^{\prime} \times 15^{\prime}\right)$ and slit spectra at two positions in the north. In this work, we present deep flux calibrated images in $\mathrm{H} \alpha+[\mathrm{N} I],\left[\mathrm{S}_{\mathrm{II}}\right]$, and additionally [O III] of the whole field around the remnant $\left(70^{\prime} \times 70^{\prime}\right)$ along with long-slit spectra at five different positions. The low ionization wide field optical images show the diffuse and patchy nature of the emission (see also Long et al. 1991). However, the presence of $\mathrm{H}$ II regions in the area does not allow the identification of emission, associated to the remnant, based solely on morphological arguments. The ratio of the $\left[\mathrm{S}_{\mathrm{II}}\right]$ to $\left.\mathrm{H} \alpha+{ }_{[\mathrm{N}} \mathrm{II}\right]$ images reveals two very interesting features. The first refers to an extended front of enhanced sulfur to $\mathrm{H} \alpha+\left[\mathrm{N}_{\mathrm{II}}\right]$ ratio running roughly in the east-west direction, while the second involves the likely detection of shock heated emission well outside the fields observed by Long et al. (1991).

It can be seen in Fig. 5 (left) that the sulfur front is in excellent positional agreement with radio, infrared and molecular emission. Since radiation at these wavelengths is produced under different conditions, this correlation further supports the interaction of the primary shock wave with interstellar molecular clouds. The south boundaries of the sulfur front and the 


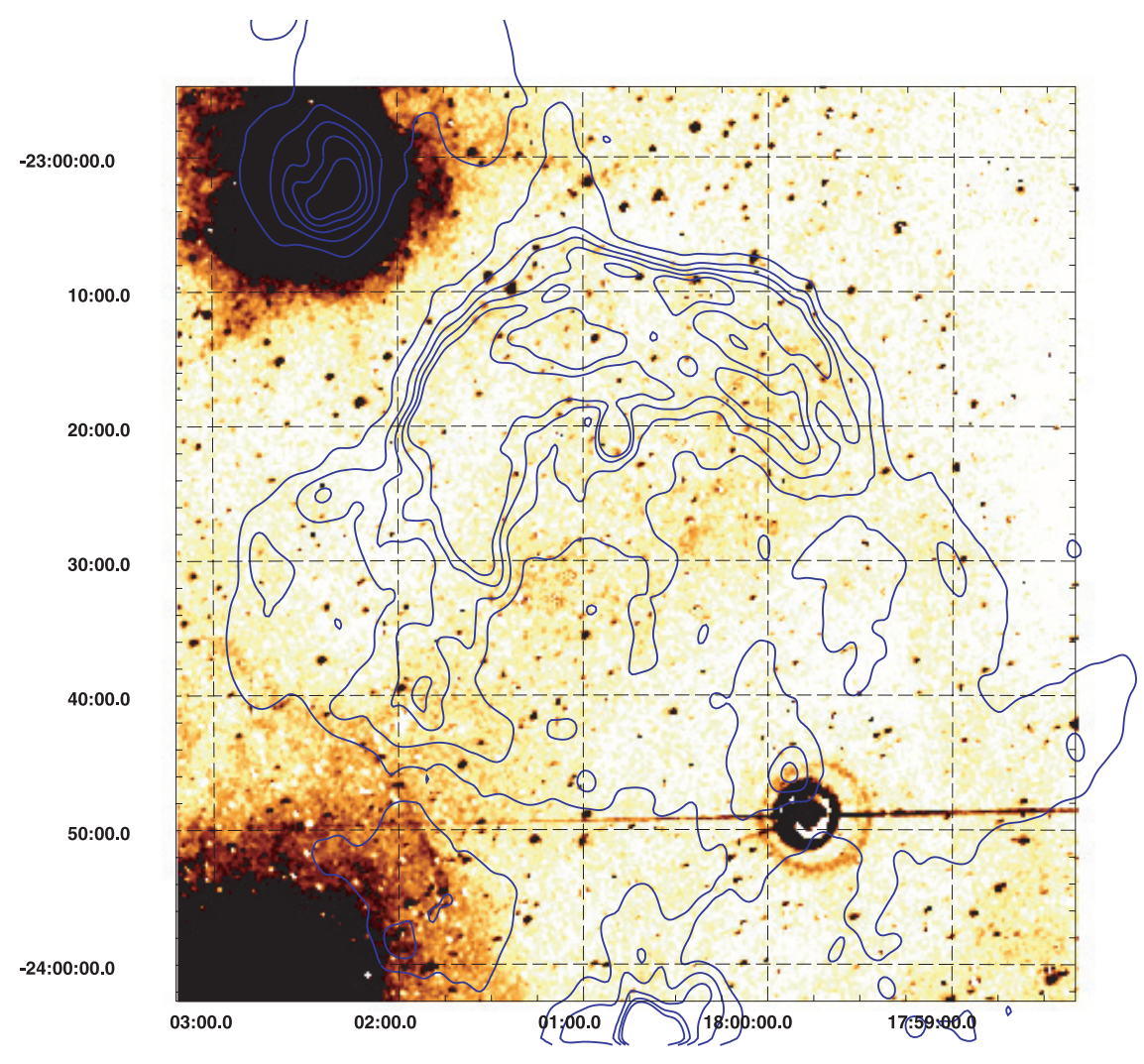

Fig. 3. Very weak emission is detected in the medium ionization line of [O III] $] 5007 \AA$. Another characteristic property of the remnant in this line is the absence of any filamentary structure. The emission is diffuse and roughly concetrated in the central areas of the remnant where fluxes around $5 \times 10^{-17} \mathrm{erg} \mathrm{s}^{-1} \mathrm{~cm}^{-2} \operatorname{arcsec}^{-2}$ are measured. Radio contours at $1.4 \mathrm{GHz}$, scaling linearly, from 0.1 to $0.54 \mathrm{Jy} \mathrm{beam}^{-1}$ are also shown. The shadings run linearly from 0 to $15 \times 10^{-17} \mathrm{erg} \mathrm{s}^{-1} \mathrm{~cm}^{-2} \operatorname{arcsec}^{-2}$.

molecular emission trace nicely the interface between the intense and the weaker emission seen in the $\mathrm{H} \alpha+\left[\mathrm{N}_{\text {II }}\right]$ image, around a declination of $-23^{\circ} 18^{\prime}$. Typically, the $\mathrm{H} \alpha+[\mathrm{N}$ II $]$ or [S II] fluxes immediately to the south of the molecular emission are stronger by a factor of $\sim 3$ than the fluxes measured over the area of $\mathrm{CO}$ emission. It may be that the molecular emission originates from an area lying towards the side of the observer, thus blocking the optical emission or that the primary shock wave has encountered a very dense cloud, as indicated by the $\mathrm{CO}(J=3-2)$ emission, driving a slower radiative shock into it. To a first order, information on this area can be extracted from our spectrum taken in the west (Pos. IVa; Table 3) which intersects the sulfur front. Examination of the models of Hartigan et al. (1994) shows that in Pos. IV we may be observing emission from a slow shock (e.g., $40-50 \mathrm{~km} \mathrm{~s}^{-1}$ ), a low ionization fraction, and a magnetic field $\sim 30-100 \mu \mathrm{G}$, for a preshock cloud density of $100 \mathrm{~cm}^{-3}$. Their model runs were performed for preshock densities of 100,1000, and $10000 \mathrm{~cm}^{-3}$. Over the area of $\mathrm{CO}$ emission we could then expect a similar or a lower shock velocity and ionization fraction but since a higher preshock density is expected, the magnetic field indicated by the models can be as high as $300 \mu \mathrm{G}$. Higher densities can be expected since $\mathrm{CO}(J=3-2)$ emission is observed in this specific area (e.g., Arikawa et al. 1999), while the estimated magnetic field is in fair agreement with that of $200 \mu \mathrm{G}$ reported by Claussen et al. (1997), determined from $\mathrm{OH}$ measurements.
We note here that the spectrum from Pos. V indicates significant $\left[\mathrm{N}_{\mathrm{II}}\right]$ emission, although the strengths of the $\left[\mathrm{N}_{\mathrm{I}}\right]$ and $\left[\mathrm{S}_{\mathrm{II}}\right]$ lines would point to low ionization. It is possible that along the sulfur front the primary shock wave encountered a dense molecular cloud allowing only for a very slow radiative shock to propagate into the cloud.

The low ionization images show that there is a significant offset between strong $\mathrm{H} \alpha+[\mathrm{N}$ II] and [S II] emission (inset of Fig. 1). The measured angular offset is equivalent to a length scale of $\sim 0.2 \mathrm{pc}$ for a distance of $1.8 \mathrm{kpc}$. This scale is too long to be accounted for by a single shock structure. For example, a spatial offset between the [O III] and $\mathrm{H} \alpha$ lines is expected according to shock models but still, may be hard to detect (e.g., Cox 1972). The imaging observations show that the $\mathrm{H} \alpha+\left[\mathrm{N}_{\text {II }}\right]$ emission is significantly reduced in a direction perpendicular to the front, while the [S II] emission is only slightly reduced. The work of Hartigan et al. (1994, their Fig. 15) shows that for a given shock velocity, a decrease in the medium density would be required in order to suppress the $\mathrm{H} \alpha$ production. On the other hand, in case of a constant preshock cloud density, the $\mathrm{H} \alpha$ flux can be suppressed by a decrease in the shock velocity. It is not clear which of the two cases holds but in reality, a combination of the two may be actually taking place. We cannot estimate the theoretical spatial offset based on the modelling of Hartigan et al. (1994) since they mainly examine the effects of shock velocity on certain optical line ratios. The calculation 


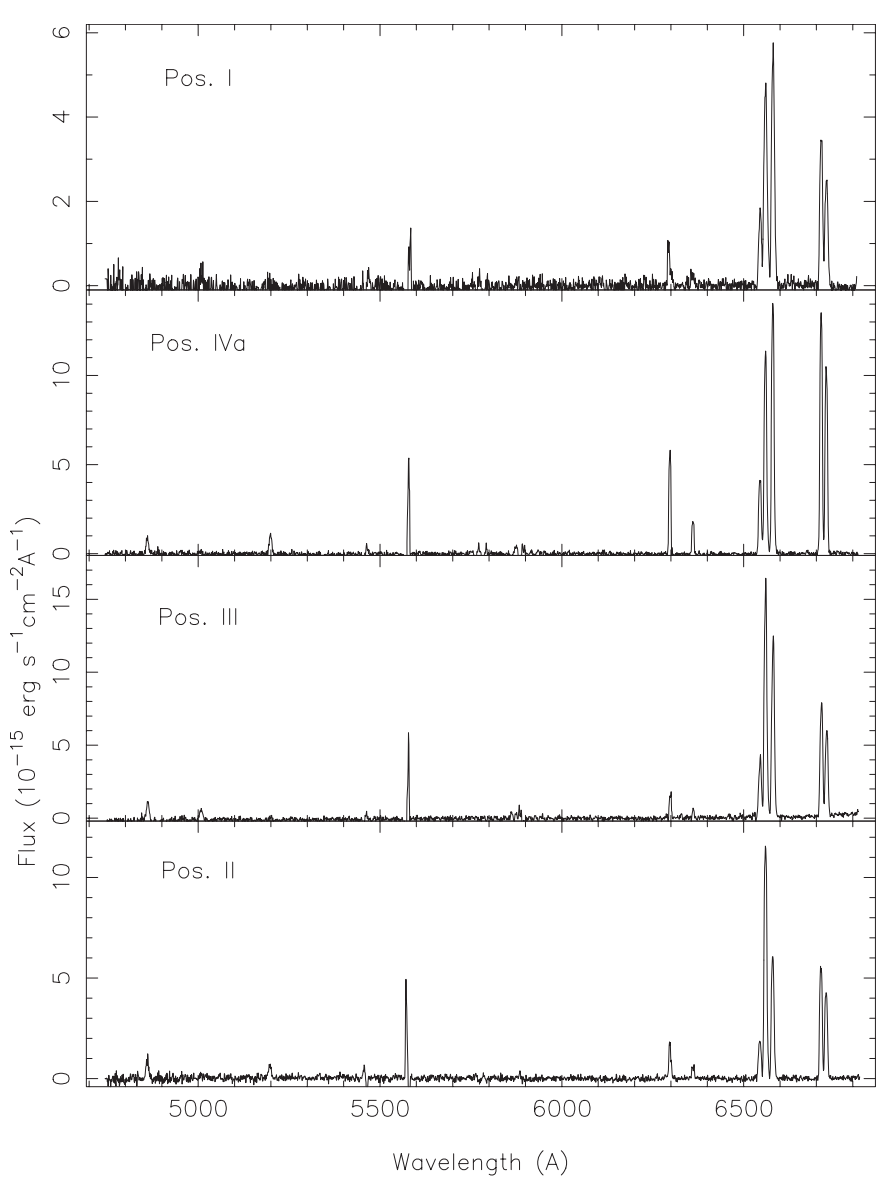

Fig. 4. Typical, observed spectra are shown in this figure from various positions of G 6.4-0.1 listed in Table 3. The flux is measured in units of $10^{-15} \mathrm{erg} \mathrm{s}^{-1} \mathrm{~cm}^{-2} \AA^{-1}$.

of the offset is further complicated by uncertain factors like e.g. multiple shock structures, and/or the viewing geometry. High resolution Echelle spectra and molecular CO observations in this area would allow us to understand the nature of the enhanced $\left[\mathrm{S}_{\mathrm{II}}\right] / \mathrm{H} \alpha+\left[\mathrm{N}_{\mathrm{II}}\right]$ ratio.

The locations where emission from shock heated gas is probably detected are marked as Pos. I and Pos. II in Fig. 2 and are located in the south-east and south-west, where no optical emission associated to the remnant was known before. The estimated $\left[\mathrm{S} \mathrm{II}_{\mathrm{II}} / \mathrm{H} \alpha\right.$ ratio at these positions is $\sim 0.7$, while the same ratio immediately to the south of e.g. Pos. I is $\sim 0.35$ pointing to an $\mathrm{H}_{\text {II }}$ region. If this identification is correct, then it implies that absorption is not limiting our view to the southwest of G 6.4-0.1 and that the primary blast wave breaking out here drives radiative shocks into the interstellar medium (ISM) clouds. It is also clear that the ISM is far from uniform in this part of the sky. In addition, dust emission at 60 and $100 \mu \mathrm{m}$ has been detected along the west boundary of G 6.4-0.1 (Fig. 5) and appears partially correlated with the radio emission of the remnant. However, it is not clear if this correlation marks the presence of an $\mathrm{HI}$ shell, which would suggest that the remnant has reached the snow-plow stage of its evolution in the west, or is a chance coincidence. Deeper and better resolution infrared observations would help to resolve this issue.
The spectra acquired at several areas of the remnant allow us to obtain a more complete view of its optical properties and the environment is interacting with. Some major spectral line ratios are given in Table 3 and these do not reveal any strong correlation with the nature of the measured radiation (e.g. diffuse; patchy). The neutral nitrogen line at $5200 \AA$ is not detected in all spectra suggesting possible changes in the ionization state of the preshocked gas. The $\left[\mathrm{N}_{\text {II] }}\right.$ emission is relatively strong ranging from 0.7 to 1.6 of the $\mathrm{H} \alpha$ flux. Interestingly, the sulfur line ratios in all positions are found close to 1.3 suggesting low postshock densities, while variations are observed in the strength of the sulfur lines relative to $\mathrm{H} \alpha$. The former remark may point to low densities of the preshocked clouds found in the ISM, or to sufficiently strong magnetic fields that can stretch the recombination zone, while the latter may also reflect changes in the ionization state of the preshocked gas. Nevertheless, UV spectra would be needed to accurately establish the ionization state of the preshocked gas and explore the physical conditions in more detail as Raymond et al. (1988) have done for a bright filament in the Cygnus Loop. The $[\mathrm{O} \mathrm{III}] / \mathrm{H} \beta$ ratios are quite low ( $\sim 1$ or less), however, this does not necessarily imply radiative shocks with complete recombination zones. Raymond et al. (1988) have shown that for complete shock structures and shock velocities between $\sim 80-140 \mathrm{~km} \mathrm{~s}^{-1}$, the $\left[\mathrm{O}_{\mathrm{III}}\right] / \mathrm{H} \beta$ does not exceed the value of $\sim 6$. However, for shock velocities below $80 \mathrm{~km} \mathrm{~s}^{-1}$, we cannot rely on the $[\mathrm{O} \mathrm{III}] / \mathrm{H} \beta$ ratio to determine the completeness or incompleteness of the recombination zone since the [O III] flux is very much reduced anyway. The overall characteristics of the optical spectra taken in the outer areas of the remnant can be accounted for by relatively slow shocks $\left(\sim 70 \mathrm{~km} \mathrm{~s}^{-1}\right)$ moving into low density and highly ionized ISM clouds. In cases of negligible magnetic fields, the relation given by Fesen \& Kirshner (1980) allows us to estimate preshock cloud densities of the order of $10 \mathrm{~cm}^{-3}$, for postshock electron densities in the range of $100-150 \mathrm{~cm}^{-3}$. The preshocked thin gas has all of its hydrogen ionized by photons coming from the X-ray gas and from the shock front. In favor of the higher ionization state of the gas in these areas is the very weak or missing $\left[\mathrm{N}_{\mathrm{I}}\right] 5200 \AA$ line.

Infrared emission is also present in the area of W28 and we use both low and high resolution IR data to compare with the radio emission of the remnant. In Fig. 5 (right) the radio image of the remnant along with contours of the $8.28 \mu \mathrm{m}$ data and HiRes IRAS $60 \mu \mathrm{m}$ data are shown (Aumann et al. 1990). Dust is clearly associated with the $\mathrm{H}$ in regions M 8 and M 20, while enhanced emission, both in 8.28 and 60 microns is seen around $\alpha \simeq 18^{\mathrm{h}} 01^{\mathrm{m}} 15^{\mathrm{s}}$ and $\delta \simeq-23^{\circ} 17^{\prime}$ extending for $\sim 6^{\prime}$ in the east-west direction. This is the area where molecular emission and strong radio, filamentary emission have been observed. The arc-like structure is also present in the $100 \mu \mathrm{m}$ HiRes IRAS data (not shown here). Although, the MSX data do not show strong emission in the west, the IRAS $60 \mu \mathrm{m}$ data reveal extended emission, of low surface brightness, running roughly in the south-north direction. Part of this emission appears to delineate the west outermost radio emission rather well, although it does not form a closed loop. 

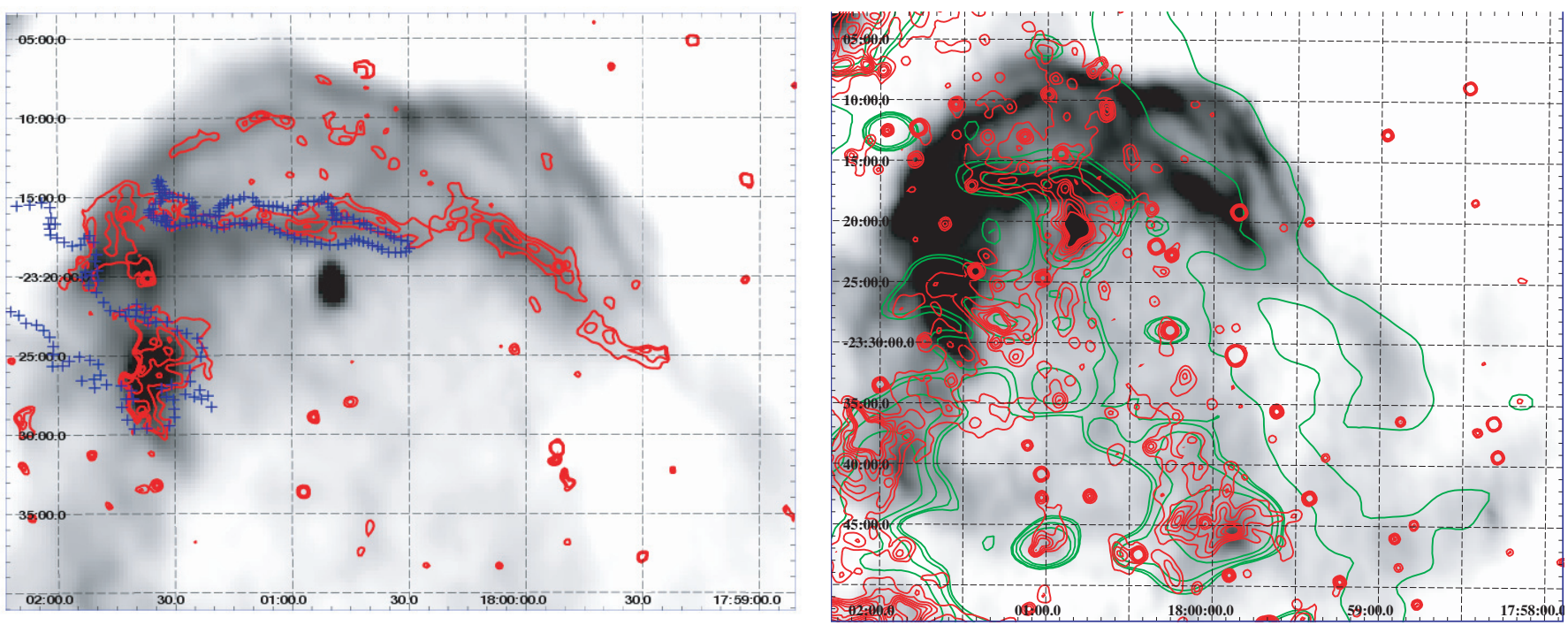

Fig. 5. The radio image at $1400 \mathrm{MHz}$ is shown in the left figure with contours outlining the front of enhanced $\left[\mathrm{S}_{\text {II }}\right] / \mathrm{H} \alpha+[\mathrm{N}$ II $]$ ratio. The crosses mark the boundary of the molecular emission detected by Arikawa et al. (1999). It is clear that the low ionization front is very well correlated with the radio emission. The radio image of G 6.4-0.1 is shown again in the right figure along with contours of the high resolution MSX data at $8.28 \mu \mathrm{m}$ (darker contours) and the lower resolution IRAS data at $60 \mu \mathrm{m}$ (lighter contours).

Usually but not always pressure equilibrium is achieved between the shocked clouds and the ISM. In order to check if pressure equilibrium does hold, we try to estimate the preshock intercloud medium density and compare it with the observations. Our long-slit spectra were taken in areas characterized by softer X-ray emission as the hardness ratio map of ROSAT PSPC data suggest (cf. Fig. 7 of Rho \& Borkowski 2002). We adopt a temperature of $\sim 0.6 \mathrm{keV}$ as representative of the temperature of the hot gas in the central to north areas of G 6.4-0.1. For a preshock cloud density of $\sim 10 \mathrm{~cm}^{-3}$ and shock velocities $\sim 50-70 \mathrm{~km} \mathrm{~s}^{-1}$, the intercloud density is estimated $\sim 0.1 \mathrm{~cm}^{-3}$, consistent with the density measured through the emission measure in the center and the north-east shell. It is clear that the optical data cannot discriminate between the adiabatic and the radiative snow-plow phases but it appears that the $\mathrm{X}$-ray gas is in pressure equilibrium with the optically emitting gas.

\section{Conclusions}

The low ionization images show the patchy nature of the emission from the remnant in its full extent. They also provide evidence that emission in the south, well outside the bulk of the known emission, may be associated to the remnant suggesting that the primary shock wave is driving radiative shocks into these clouds. However, the failure to detect more emission from shock heated gas in the south could imply a tenuous interstellar medium incorporating much denser clouds. The $\mathrm{H} \alpha+\left[\mathrm{N}_{\mathrm{II}}\right]$ and [S II] images are used to identify a strong sulfur front extending roughly in the east-west direction. Its central and eastern parts are very well correlated with $\mathrm{CO}$ emission indicating that we observe emission coming from a slow shock travelling into a largely neutral molecular cloud. The low degree of ionization of the preshocked clouds is also supported by the detection of adequate [N I $] 5200 \AA$ emission. The long-slit spectra obtained in the perimeter of the remnant imply shock velocities around
$70 \mathrm{~km} \mathrm{~s}^{-1}$ and low preshock cloud densities $\sim 10 \mathrm{~cm}^{-3}$, in case of low magnetic fields. Finally, the medium ionization line image in [O III] reveals very weak diffuse emission in the central to west part of the remnant, while in the east, the level of the emitted flux is below our detection limit. This suggests that the low shock velocities driven into the interstellar clouds are a global characteristic of W28 and its environment.

Acknowledgements. The authors would like to the referee for stimulating comments which helped to improve and clarify the scope of this work. We would also like thank N. Kylafis and J. Rho for useful comments and G. Dubner for providing the $1.4 \mathrm{GHz}$ image of W28. Skinakas Observatory is a collaborative project of the University of Crete, the Foundation for Research and Technology-Hellas and the Max-Planck-Institut für Extraterrestrische Physik. This research has made use of data obtained through the High Energy Astrophysics Science Archive Research Center Online Service, provided by the NASA/Goddard Space Flight Center.

\section{References}

Arikawa, Y., Tatematsu, K., Sekimoto, Y., \& Takahasi, T. 1999, PASJ, 51, L7

Aumann, H. H., Fowler, J. W., \& Melnyk, M. 1990, AJ, 99, 1674

van den Bergh, S., Marscher, A. P., \& Terzian, Y. 1973, ApJS, 227, 26

Bohigas, J., Ruíz, M. T., Carrasco, L., Salas, L., \& Herrera, M. A. 1983, Rev. Mex. Astron. Astrofis., 8, 155

Claussen, M. J., Frail, D. A., Goss, W. M., \& Gaume, R. A. 1997, ApJ, 489,143

Cox, D. P. 1972, ApJ, 178, 143

Dopita, M. A., Mathewson, D. S., \& Ford, V. L. 1977, ApJ, 214, 179

Dubner, G. M., Velázquez, P. F., Goss, W. M., \& Holdaway, M. A. 2000, ApJ, 120, 1933

Esposito, J. A., Hunter, S. D., Kanbach, G., \& Sreekumar, P. 1996, ApJ, 461, 820

Frail, D. A., \& Mitchell, G. F. 1998, ApJ, 508, 690

Fesen, R. A., \& Hurford, A. P. 1995, AJ, 110, 747

Fesen, R. A., \& Kirshner, R. P. 1980, ApJ, 242, 1023 
Hamuy, M., Walker, A. R., Suntzeff, N. B., et al. 1992, PASP, 104, 533 Hamuy, M., Suntzeff, N. B., Heathcote, S. R., et al. 1994, PASP, 106, 566

Hartigan, P., Raymond, J., \& Hartmann, L. 1987, ApJ, 316, 323

Hartigan, P., Morse, J. A., \& Raymond, J. 1994, ApJ, 436, 125

Hunter, D. A. 1992, ApJS, 79, 469

Yusef-Zadeh, F., Shure, M., Wardle, M., \& Kassim, N. 2000, ApJ, 540, 842

Kaspi, V. M., Lyne, A. G., Manchester, R. N., et al. 1993, ApJ, 409, L57

Kundu, M. R. 1970, ApJ, 162, 17

Lasker, B. M., Russel, J. N., \& Jenkner, H. 1999, in the HST Guide Star Catalog, version 1.1-ACT, The Association of Universities for Research in Astronomy, Inc.
Long, K. S., Blair, W. P., Matsui, Y., \& White, R. L. 1991, ApJ, 373, 567

Mavromatakis, F., Boumis, P., \& Goudis, C. 2003, A\&A, 405, 591

Milne, D. K., \& Hill, E. R. 1969, AuJPA, 22, 211

Milne, D. K., \& Wilson, T. L. 1971, A\&A, 10, 220

Raymond, J. C., Hester, J. J., Cox, D., et al. 1988, ApJ, 324, 869

Rho, J., \& Borkowski, K. J. 2002, ApJ, 575, 201

Shaver, P. A., \& Goss, W. M. 1970, AuJPA, 14, 77

Shaw, R. A., \& Dufour, R. J. 1995, PASP, 107, 896

Smith, R. C., Kirshner, R. P., Blair, W. P., Long, K. S., \& Winkler, P. F. 1993, ApJ, 407, 564 